\title{
Development of Fixed Device for Rotating Ultrasonic Sensor
}

\author{
Bo Zhu, Hua Zhang, Xinnan Xie, Chenghao Lan, Shuyi Tao, Miao Wang, \\ Tianshu Hai, Yanjun Pang, Qinghao Wang \\ Fushun Power Supply Company, Liaoning Electric Power Company Limited, State Grid, China
}

Keywords: GIS; Ultrasonic partial discharge; Sensor; Universal rotation; Field application

\begin{abstract}
In order to solve the problem of GIS equipment structure and limited space, the paper develops the fixed device for rotary ultrasonic sensor based on the actual situation, expounds the principle of ultrasonic testing, and introduced the partial discharge defects, corona defects, free metal particle defects can be found by testing ultrasonic partial discharge test. It discusses that the device can effectively solve the problems of the prior test GIS ultrasonic partial discharge, and ensure the safety of the test personnel.
\end{abstract}

\section{Introduction}

Gas insulated substation also known as SF6 insulated substation(GIS), is the entire substation equipment (except the transformer) all enclosed in a grounded metal shell, the shell is usually filled with $0.3-0.5 \mathrm{Mpa}$ SF6, to ensure that the ground, and the reliability of the fracture between the insulation. SF6 has the strong ability of arc extinguishing (air 100 times), high insulating capacity (air 2.9 times), it is a Non inflammable and explosive inert gas, pure SF6 is a colorless, odorless and non-toxic ${ }^{[1]}$. Because the chemical nature of SF6 is not active, it can be used as insulation to replace the previous substation with bare wire to connect all kinds of electrical equipment and the use of air as a method of insulation. GIS makes the structure and operation of substation has undergone tremendous changes, its remarkable characteristic is integration, miniaturization, beautiful and labor-saving ${ }^{[2]}$. But once an accident occurs, the consequences of the damage than the separation of the equipment is much more serious, the repair cycle of failure is long and complex.

\section{The Principle of Ultrasonic Testing}

\section{The partial discharge.}

In the insulation of power equipment, only partial discharge phenomenon and not through the voltage applied between the conductor (i.e. not breakdown) is called partial discharge. It is due to the local electric field distortion, local field strength, which caused by insulation within the scope of local gas discharge or breakdown. It may occur in the conductor on the edge, may also occur on insulator's surface or internal. The partial discharge of insulator even corrosion insulation materials, and finally lead to the breakdown of insulation. Therefore, partial discharge detection can prevent the occurrence of insulation accidents, which is great significance to the maintenance of equipment safety and stable operation of power system.

Partial discharge is a pulsed discharge, it will produce a series of light, sound, electrical and mechanical vibration and other physical and chemical changes inside and around the power equipment. These various physical and chemical changes caused by partial discharge, they can provide the detection signal for the internal monitoring of power equipment insulation condition. When the partial discharge signal is generated, the vibration and sound of the electric power equipment will be generated. The ultrasonic method is used to measure the partial discharge signal by the ultrasonic sensor mounted on the external wall of the equipment. The characteristic of this method is that there is no connection between the sensor and the electric circuit of the power equipment. It is not affected by the electrical disturbance, but it is easy to be affected by the ambient noise or the mechanical vibration of the equipment when the field is used.

Ultrasonic wave is the elastic wave of frequency greater than $20 \mathrm{kHz}$, the frequency of GIS ultrasonic testing is generally selected $20 \sim 80 \mathrm{kHz}$, The ultrasonic test is carried out under the 
condition of GIS. It can be found in a timely manner in the presence of a variety of GIS anomalies or fault omen, so as to make timely treatment, and prevent high density adjacent components from spreading $^{[1]}$.

Ultrasonic partial discharge test can detect partial discharge defects, corona defects, free metal particle defects.

(1)The partial discharge defects

This kind of defect is a non penetrating discharge in the equipment. It is mainly caused by the suspended electrode (i.e., non grounded and high pressure metal), internal air bubbles, and the surface contamination. This kind of defects and the power frequency electric field have obvious correlation, it is the main threat to the breakdown of equipment insulation, should focus on testing.

(2)The corona defects

This kind of defect is mainly caused by the inner conductor of the equipment, the burr of the shell, etc., It is mainly manifested as a single discharge phenomenon for the conductor to the surrounding medium (such as SF6) .This kind of defect is less harmful to the equipment, but still exists equipment breakdown hidden danger under the action of over voltage, and should be concerned based on the magnitude of the signal.

(3) The free metal particle defects

This kind of defects mainly exists in GIS, which is mainly caused by the metal debris generated by the equipment installation process or the switch action process. With the periodic change of internal electric equipment, this kind of metal particles has random moving or jumping phenomenon, When the particle's jump amplitude between the high voltage conductor and the low pressure casing. increases, there is a risk of equipment breakdown, should be paid more attention to.

\section{Steps for Ultrasonic Testing of GIS.}

GIS ultrasonic partial discharge detection usually has the following two kinds of problems:

(1)The distance between the gas chamber and the ground is high. Due to the Cylinder type structure of GIS. It is no suitable point of the safety belt when climbing test, especially in winter, the equipment shell is more slippery, these test points are ignored for security reasons sometimes.

(2) The GIS has the compact device structure and narrow space. For example, the adjacent GIS equipment is too close to the bottom of the gas chamber while two testers are required to drill into the chamber when testing the B phase circuit breaker. It is easy to appear personal equipment, bump phenomenon, also brings inconvenience to the testing work

\section{Device Design Scheme.}

In view of the above problems, we have developed the following fixed device,it is similar to mechanical hands and can rotate ultrasonic sensors. The device is mainly composed of three parts, which is retractable spear, universal rotation and sensor head.

Sensor head.

The sensor is placed in a porcelain shell cover, the compression springs are under the sensor., the top cover porcelain magnet. The top of the porcelain housing is equipped with a ceramic magnet. The magnet makes internal spring compression through adsorbing the surface of the measured object and fixes the sensor. Connecting line between sensor and host passes through the retractable spear. The following figure is a structure diagram:

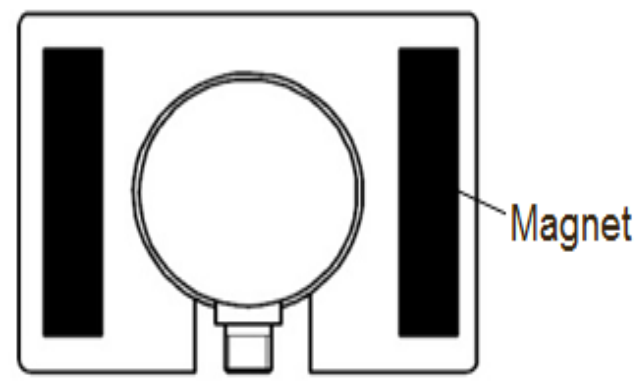

Vertical view

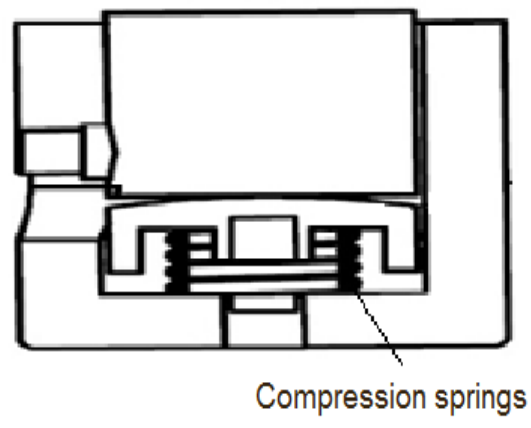

Front elevation

Figure 1. Schematic diagram of the sensor 


\section{Universal Rotation.}

The core structure of universal rotation is the ball head and the adjusting nut. the ball head is connected to the sensor head, this can ensure that the sensor can be rotated in multi direction and multi angle, and Adjusting nut is used to adjust the flexibility of sensor rotation. As shown in Figure 2 .

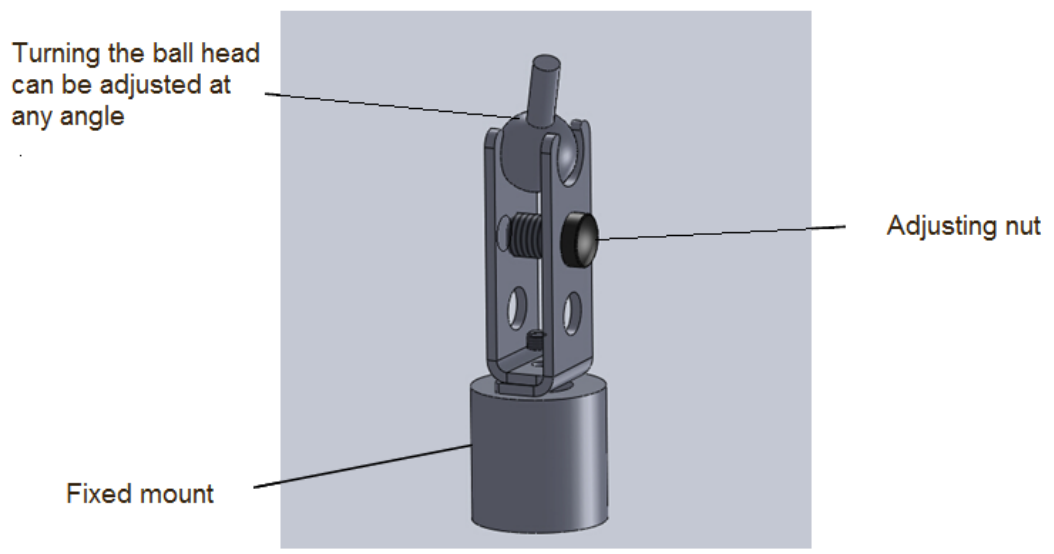

Figure 2. Schematic diagram of universal rotation

Retractable Spear.

The multi section retractable spear can be stretched or contracted to the specified length when the device is used, and then the rod can be locked and fixed in this position. As shown in Figure 3.

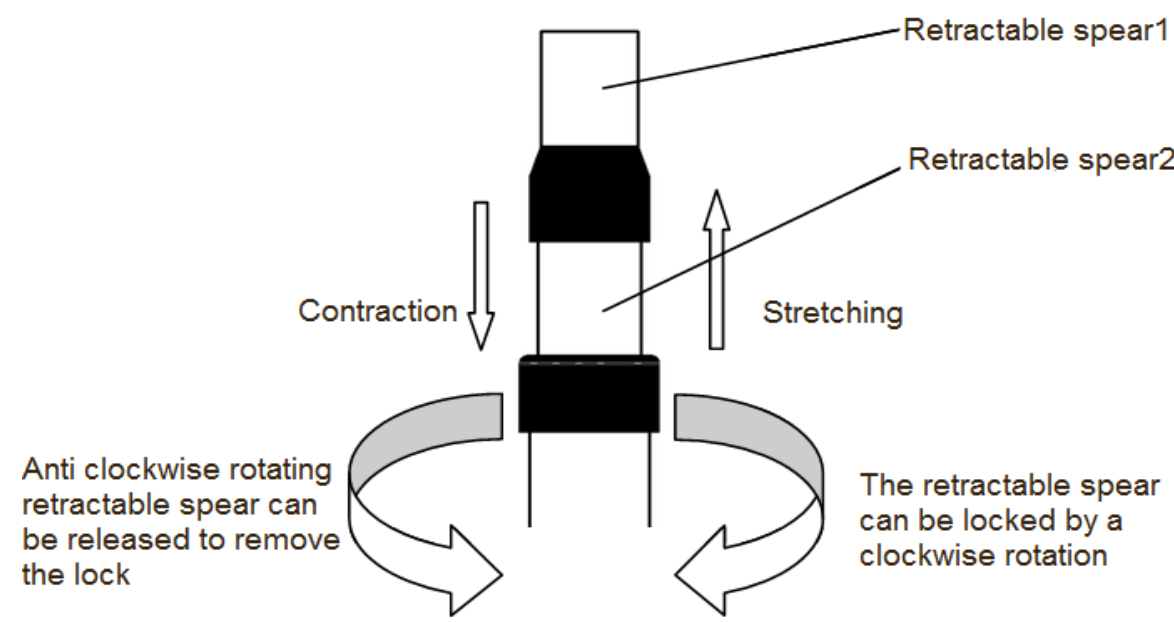

Figure 3. Schematic diagram of retractable spear

\section{The assembly drawing.}

The schematic diagram of the device after assembly is shown in Figure 4.
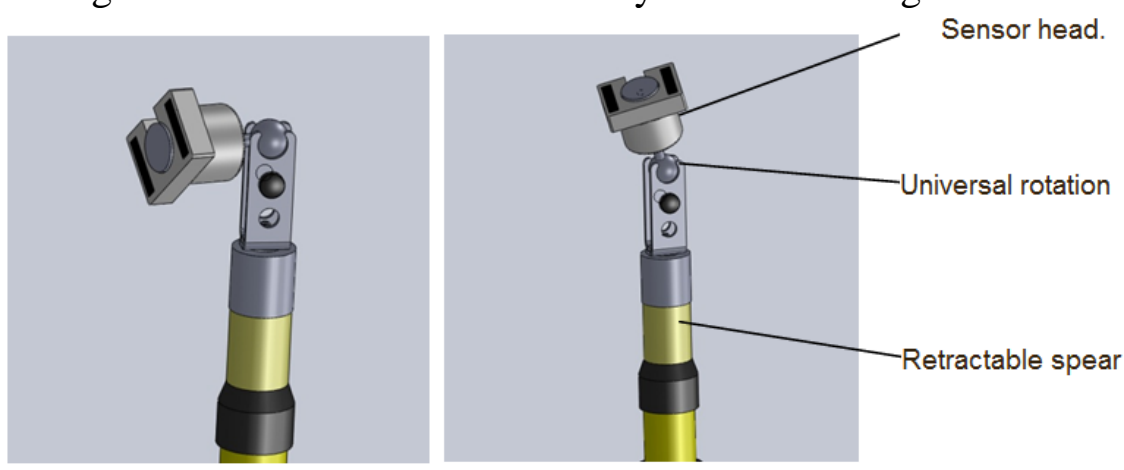

Figure 4. The schematic diagram after assembly 


\section{Instructions.}

(1) The sensor does not rotate due to its own gravity through adjusting the adjusting nut of the universal rotary part, but excessive regulation will make the sensor unable to adsorb on the measured point of GIS automatically.

(2) According to the height of the GIS's measuring point, the retractable spear of the device is adjusted to the proper position, then rotate the retractable spear in a clockwise rotation;

(3)The sensor head will reach a position near the point to be measured by retractable spear, the sensor head can be automatically adsorbed to the portion to be tested, The best is that the sensor head can rotate freely through the adsorption force(The premise is retractable spear is prohibited to move), Otherwise, the adjusting nut of the universal rotation should be adjusted properly.

(4)The main engine analysis the record of the waveform. Then follow these steps to test the next point.

\section{Field Application of the Device}

July 20, 2015, the device is applied to the partial discharge test of GIS ultrasonic in LiuLin $220 \mathrm{kV}$ substation. GIS equipment of LiuLin is compact in structure and narrow in space. When testing, the staff needs to drill into the gas chamber when testing the B phase circuit breaker of the 5 lines such as LiuZhong line and LiaoLinThree Line. The working environment is poor, the condition is difficult, it is easy to cause personal injury and equipment damage accident. Staff use "fixed device for rotating ultrasonic sensor" for this testing. Staff do not have to drill into the chamber below, to avoid knocking people and equipment etc. So that it can be fast and accurate, safe and reliable to complete the test work.

In addition, the device is applied to the partial discharge test of GIS ultrasonic in NanYang 66kV substation. Because the air chamber of the 9 lines in NanYang is very high, normal testing needs the high operation, and could not find a suitable point for hanging safety belt, and it is easy to happen personal injury accident such as falling and slipping. Staff use "fixed device for rotating ultrasonic sensor" for this testing, it avoids possible problems, also can be fast and accurate, safe and reliable to complete the test work.

\section{Conclusions}

The fixed device for rotating ultrasonic sensor has the following advantages. It is not limited by the equipment structure and space of GIS; It does not exist in the high work, can ensure the safety of the test personnel; it can be arbitrarily selected test points and ensure accurate data; Lead in the retractable spear is not easy to damage; this device is easy to use, and solves the practical problems, so it can be applied to the field work. It can know that the whole idea is correct, clear, good application effect, and has a wide range of promotional value and application prospects through practical work.

\section{References}

[1] Zhou Zecun. High voltage technology. Water conservancy and electric power press. 1996.12

[2] Chen Huagang. Preventive test method and diagnosis technology of electric power equipment [M]. Beijing science and Technology Press.2001

[3] Chen Yuxiang. Electrical test. China Electric Power Press.2016.1 